\title{
Sostenibilidad. Hemodiálisis frente a diálisis peritoneal
}

\author{
Eduardo Garoé Fernández García
}

Enfermero

Fundación Hospital Calahorra. Logroño. La Rioja

\section{Sr. Director:}

La situación económico - sanitaria actual no es halagüeña y ha sido la que nos ha motivado en el desarrollo del presente estudio. Términos como crisis y sostenibilidad se han hecho de gran actualidad, difundiéndose en los medios de comunicación ${ }^{1,2}$.

El diccionario de la Real Academia de la Lengua Española define estos términos de la siguiente manera:

- Crisis: 6. f. Escasez, carestía; 7. f. Situación dificultosa o complicada.

- Sostenible: Dicho de un proceso, que puede mantenerse por sí mismo, como lo hace, p. ej., un desarrollo económico sin ayuda exterior ni merma de los recursos existentes.

Inmersos en esta situación, el desconocimiento sobre el verdadero coste económico sanitario, por parte tanto del profesional sanitario como del usuario ${ }^{3,4}$, no ayuda en el impulso de medidas que potencien un cambio positivo hacia la máxima sostenibilidad posible del sistema sanitario, algo posible con esfuerzos integrados, como se refiere en el Informe: Desarrollo Autonómico, Competitividad y Cohesión Social en el Sistema Sanitario del Consejo Económico y Social, de Marzo de $2011^{5}$.

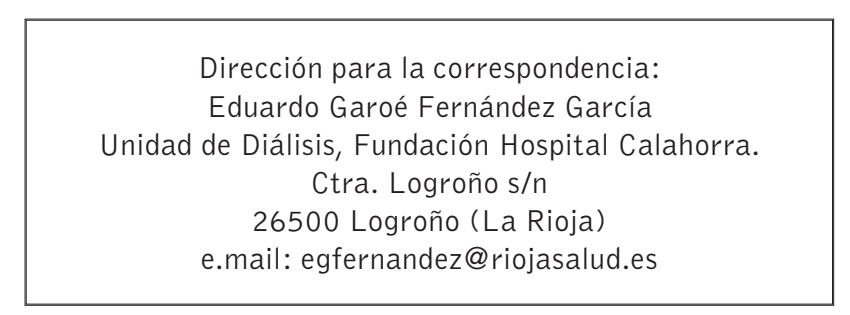

Desde el punto de vista nefrológico siempre nos ha preocupado el coste generado por la especialidad. En 2006 desarrollamos un estudio para evidenciar que técnicas de hemodiálisis eran económicamente más costosas ${ }^{4}$.

En líneas generales es conocido por parte de los profesionales sanitarios nefrológicos el mayor coste general de la hemodiálisis (HD) frente a la diálisis peritoneal (DP), pero ¿de qué cantidades estamos hablando? y ¿realmente nos puede ayudar este conocimiento en la elección de una alternativa terapéutica u otra para un paciente determinado?

Nuestro objetivo, fue por tanto, analizar el coste comparativo entre las dos alternativas nefrológicas, HD y DP, existentes en nuestro hospital, en el año 2010.

\section{Material y método}

La FHC es un hospital comarcal, referencia sanitaria para unos 80.000 habitantes.

En 2010-2011 nuestra unidad proporciona tratamiento dialítico a 40 pacientes, 4 de ellos en diálisis peritoneal automatizada y 36 en hemodiálisis, aunque se admiten pacientes desplazados que forman parte de la estadística. (Figura 1)

La dotación de personal es de tres F.E. en Nefrología (la actividad de uno de los cuales está distribuida entre un $85 \%$ en HD y un $15 \%$ en DP), seis D.U.E (la actividad de uno de los cuales está distribuida entre 80 $\%$ en HD y un $20 \%$ D.P) , 4 T.C.A.E y 1 Coordinador de enfermería. (Figura 2). 


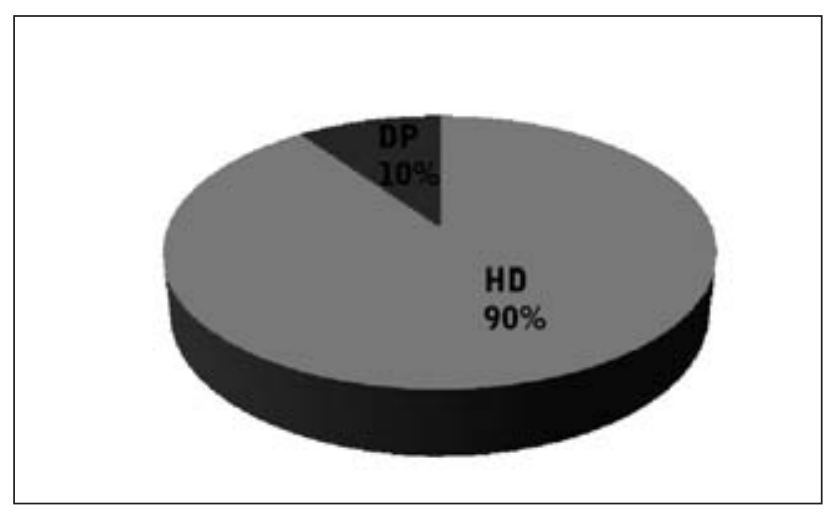

Figura 1. Distribución pacientes HD vs DP



Figura 2. Distribución de profesionales

A lo largo de 2010 se realizaron 5393 sesiones de HD y 1107 sesiones en DP (días de tratamiento). (Figura 3).

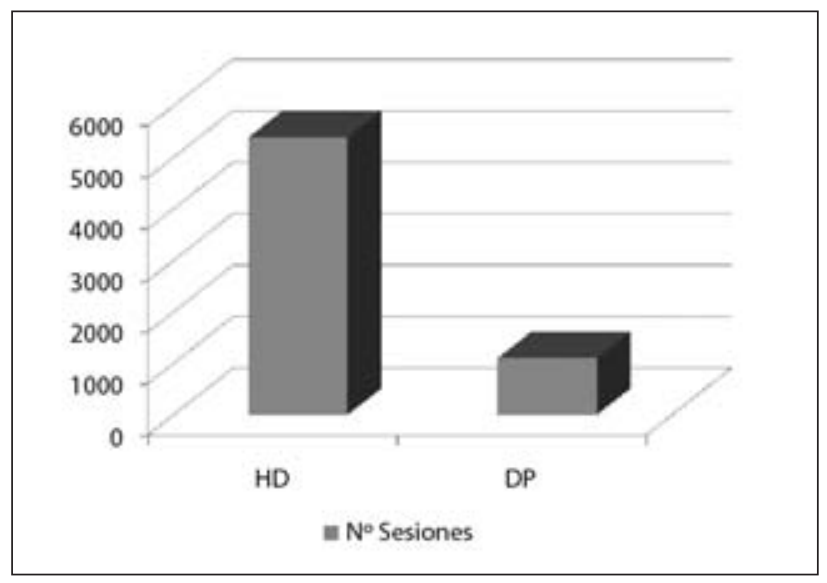

Figura 3. Sesiones HD vs Sesiones DP 2010
Hemos hecho un análisis económico, comparativo de las dos técnicas depurativas disponibles en nuestro hospital.

Para analizar el coste derivado de la actividad de hemodiálisis, usamos la misma metodología empleada en el estudio desarrollado en 20064, adaptándolo al año 2010 y por medio del cual llegamos a conocer el coste de las distintas técnicas de HD utilizadas en nuestra unidad así como el impacto global económico en el hospital, de esta alternativa terapéutica.

En cuanto al coste generado por la actividad de diálisis peritoneal, se obtuvieron las facturas derivadas de los tratamientos de los pacientes, a lo largo de todo el año 2010 prorrateando el gasto por cada uno de ellos.

Ambas áreas terapéuticas tienen un enfoque desde la perspectiva de eficacia y eficiencia, con base en los datos de actividad y contabilidad de costes, a través del cuadro de mando integral de la organización. Mediante un grupo de trabajo (taller Kaizen), desarrollado por la Unidad de Contabilidad y la Unidad de Diálisis, se fijan las pautas de trabajo y los objetivos a conseguir, planificación y sistemática de cada uno de los gastos implícitos a cada una de las alternativas terapéuticas por el GRD (Grupos relacionado por el diagnóstico), dentro del GFH (Grupo Funcional Homogéneo) de la Unidad de Diálisis. En este grupo de trabajo se ha contado con el software informático GESCOT (gestión de costes) y Bussines Intelligent ( $\mathrm{BI}$, ), unas herramientas que permiten y agilizan la puesta en marcha y explotación de los datos económicos y asistenciales.

Fueron contempladas las siguientes áreas:

- Farmacia.

- RRHH.

- Laboratorio.

- Hostelería.

- Mantenimiento.

- Lencería.

- Gastos Varios (Teléfono, electricidad, etc.)

- Transporte ambulancias. Se realizó una estimación fuera del estudio analítico central, debido a la facturación externa de este servicio y que se contrastó con la información obtenida de otros centros. 
Se computaron todos los conceptos a cada paciente en tratamiento, asignándolos a los números de historia correspondientes y obteniendo finalmente un registro en Excel del que se extrajeron los resultados del estudio.

\section{Resultados}

Los datos obtenidos de los registros correspondientes (Tablas 1 y 2) fueron explotados y complementados en un último registro (Tabla 3), con la estimación realizada, sobre el coste derivado del transporte en ambulancia de los pacientes, que ascendía a $18 €$ de media por cada desplazamiento de ida y vuelta realizado al centro hospitalario.

\begin{tabular}{|c|c|c|c|}
\hline & Coste Total & $\begin{array}{c}\text { Cantidad } \\
\text { de Producto }\end{array}$ & $\begin{array}{l}\text { Coste Unidad } \\
\text { de Producto }\end{array}$ \\
\hline ID & $1,670,818.49$ & $5,393.00$ & 309.81 \\
\hline$X X X$ & $58,926.08$ & 159 & 370,6 \\
\hline$X X X$ & $32,668.77$ & 82 & 398.4 \\
\hline$X X X$ & $51,060.01$ & 167 & 305.75 \\
\hline$X X X$ & 917.25 & 3 & 305.75 \\
\hline$X X X$ & $47,696.77$ & 156 & 305.75 \\
\hline$X X X$ & $49,197.72$ & 177 & 277.95 \\
\hline$X X X$ & $2,779.53$ & 10 & 277.95 \\
\hline$X X X$ & $58,184.87$ & 157 & 370.6 \\
\hline$X X X$ & $1,945.67$ & 6 & 324.28 \\
\hline$X X X$ & $1,945.67$ & 6 & 324.28 \\
\hline$X X X$ & $19,262.16$ & 63 & 305.75 \\
\hline$X X X$ & $1,834.49$ & 6 & 305.75 \\
\hline$X X X$ & $41,970.94$ & 151 & 277.95 \\
\hline$X X X$ & $11,396.08$ & 41 & 277.95 \\
\hline$X X X$ & $56,702.46$ & 153 & 370.6 \\
\hline$X X X$ & $23,533.37$ & 127 & 185.3 \\
\hline$X X X$ & $52,116.23$ & 125 & 416.93 \\
\hline$X X X$ & $39,474.31$ & 130 & 305.75 \\
\hline$X X X$ & $35,578.01$ & 128 & 277.95 \\
\hline$X X X$ & $13,712.36$ & 37 & 370.6 \\
\hline$X X X$ & $58,555.48$ & 158 & 370.6 \\
\hline$X X X$ & $57,814.27$ & 156 & 370.6 \\
\hline
\end{tabular}

\begin{tabular}{|l|l|l|l|}
\hline$X X X$ & $10,376.92$ & 28 & 370.6 \\
\hline$X X X$ & $60,037.90$ & 162 & 370.6 \\
\hline$X X X$ & $43,638.66$ & 157 & 277.95 \\
\hline$X X X$ & $25,571.70$ & 92 & 277.95 \\
\hline$X X X$ & $57,814.27$ & 156 & 370.6 \\
\hline$X X X$ & $43,638.66$ & 157 & 277.95 \\
\hline$X X X$ & $37,931.35$ & 178 & 213.1 \\
\hline$X X X$ & $29,092.44$ & 157 & 185.3 \\
\hline$X X X$ & $46,404.29$ & 159 & 291.85 \\
\hline$X X X$ & $61,372.07$ & 192 & 319.65 \\
\hline$X X X$ & $42,248.89$ & 152 & 277.95 \\
\hline$X X X$ & $43,916.61$ & 158 & 277.95 \\
\hline
\end{tabular}

Tabla 1: Coste Hemodiálisis 2010 (extracto)

\begin{tabular}{|l|c|c|c|}
\hline & Coste Total & $\begin{array}{c}\text { Cantidad de } \\
\text { Producto }\end{array}$ & $\begin{array}{c}\text { Coste Unidad } \\
\text { de Producto }\end{array}$ \\
\hline ID & $84,860.58$ & $1,107.00$ & 76.65 \\
\hline XXX & $25,539.59$ & 364 & 70.16 \\
\hline XXX & $7,849.91$ & 56 & 140.6 \\
\hline XXX & $29,081.01$ & 365 & 79.67 \\
\hline XXX & $22,390.07$ & 322 & 69.53 \\
\hline
\end{tabular}

Tabla 2: Coste Diálisis Peritoneal 2010

\begin{tabular}{|c|c|c|}
\hline Tipo tratamiento & $\begin{array}{c}\text { HD } \\
\text { (156 sesiones/año) }\end{array}$ & $\begin{array}{c}\text { DP } \\
\text { (365 sesiones año) }\end{array}$ \\
\hline Coste/Unidad & 309.81 & 76.65 \\
\hline $\begin{array}{c}\text { Coste Promedio/ } \\
\text { Paciente año }\end{array}$ & $48,330.36$ & $27,977.25$ \\
\hline $\begin{array}{c}\text { Transporte } \\
\text { (18€/Desplazamiento) }\end{array}$ & 2,808 & 216 \\
\hline TOTAL & $\mathbf{5 1 , 1 3 8 . 3 6}$ & $\mathbf{2 8 , 1 9 3 . 2 5}$ \\
\hline
\end{tabular}

Tabla 3: Coste Hemodiálisis frente a diálisis peritoneal 2010

En este punto, para refinar más el análisis se hizo un desglose por estructura de coste de Hemodiálisis ( Tabla 4) y Diálisis Peritoneal (Tabla 5), de modo que se evidencia claramente en que áreas repercute el peso más importante de cada alternativa terapéutica, como se puede ver en los gráficos. (Figuras 4 y 5). 
[ E. G. Fernández García, et al ]

Sostenibilidad. Hemodiálisis frente a diálisis peritoneal

\begin{tabular}{|c|c|c|c|}
\hline & $\begin{array}{l}\text { Centro de Coste: DIA } \\
\text { HEMODIÁLISIS }\end{array}$ & 2010 & peso $\%$ \\
\hline \multirow{5}{*}{ 1. COSTES PROPIOS DE PERSONAL } & & 677.418,75 & $38.22 \%$ \\
\hline & 01 Médicos & $185.412,23$ & $10.46 \%$ \\
\hline & 02 Enfermeros & $349.770,25$ & $19.73 \%$ \\
\hline & 06 TCAE & $127.807,57$ & $7.21 \%$ \\
\hline & OTROS & $14.428,70$ & $0.81 \%$ \\
\hline \multirow{5}{*}{ 2. COSTES PROPIOS DE FUNCIONAMIENTO } & & $612.747,88$ & $34.57 \%$ \\
\hline & 60010000 Farmacia & $103.420,88$ & $5.83 \%$ \\
\hline & 60130010 Material hemodiálisis & $383.424,84$ & $21.63 \%$ \\
\hline & 60770100 Servicio alimentación & $11.445,03$ & $0.64 \%$ \\
\hline & OTROS (Material no clínico, formación, etc.) & $114.457,06$ & $6.46 \%$ \\
\hline \multirow{7}{*}{$\begin{array}{l}\text { 3. COSTES IMPUTADOS POR CENTROS DE } \\
\text { COSTE INTERMEDIOS }\end{array}$} & & $286.689,25$ & $16.18 \%$ \\
\hline & LAV Lavandería & $10.331,82$ & $0.58 \%$ \\
\hline & LIM Limpieza & $30.485,53$ & $1.72 \%$ \\
\hline & MANT Mantenimiento & $18.628,04$ & $1.05 \%$ \\
\hline & Mantenimiento electromedicina & $40.267,95$ & $2.78 \%$ \\
\hline & RESI Gestión de residuos & $49.250,65$ & $2.78 \%$ \\
\hline & OTROS (Gases medicinales, esterilización, etc) & $137.725,26$ & $7.77 \%$ \\
\hline \multirow{6}{*}{$\begin{array}{l}\text { 4. COSTES IMPUTADOS POR CENTROS DE } \\
\text { COSTE ESTRUCTURALES }\end{array}$} & & $93.962,61$ & $5.30 \%$ \\
\hline & DEF Dirección económico financiera & $23.045,81$ & $1.30 \%$ \\
\hline & MPR Medicina preventiva & $9.546,37$ & $0.54 \%$ \\
\hline & PRIE Prevención de riesgos & $1.295,04$ & $0.07 \%$ \\
\hline & RRHH Recursos Humanos & $15.183,80$ & $0.85 \%$ \\
\hline & OTROS & $44.891,59$ & $2.53 \%$ \\
\hline 5.TRANSPORTE & Transporte & $101,443.66$ & $5.72 \%$ \\
\hline TOTAL & & $1.772 .262,15$ & \\
\hline
\end{tabular}

Tabla 4: Estructura de Coste Hemodiálisis

\begin{tabular}{|l|l|l|l|}
\hline & \multicolumn{1}{|c|}{$\begin{array}{c}\text { Centro de Coste: DIA } \\
\text { D. PERITONEAL }\end{array}$} & 2010 & peso \% \\
\hline 1. Costes propios de personal & RRHH & 3978,62 & $4,63 \%$ \\
\hline 2. Costes propios de funcionamiento & Facturación & 78262,66 & $91,29 \%$ \\
\hline 3. Costes imputados por centros de coste intermedios & Laboratorio & 2619,3 & $3,05 \%$ \\
\hline 4. Transporte & Transporte & 864.00 & $1.00 \%$ \\
\hline
\end{tabular}

Tabla 5: Estructura de Coste Diálisis Peritoneal 


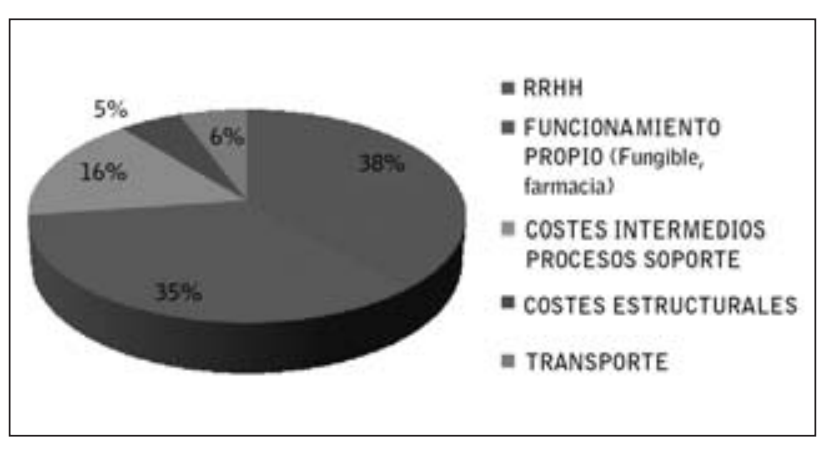

Figura 4. Estructura de Costes HD 2010

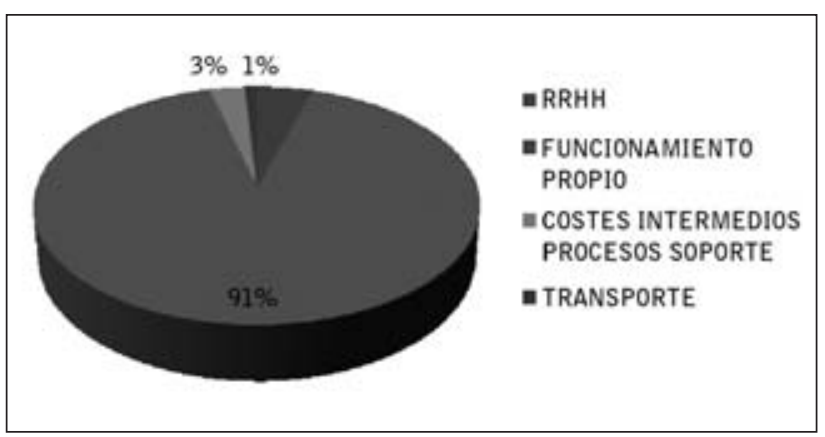

Figura 5. Estructura de Costes DP 2010

Observamos que la estructura de coste de hemodiálisis es prácticamente una constante como en el estudio desarrollado en $2006^{4}$.

Ante estos resultados se nos plantea la siguiente discusión:

Si la diferencia de coste es casi la mitad a favor de la $\mathrm{DP}, \mathrm{y}$ teniendo en cuenta el gran número de pacientes que reciben tratamiento extrarenal en nuestro país, cabe pensar que una medida dirigida hacia la sostenibilidad del sistema sanitario podría ser la inclusión de un mayor número de pacientes en programas de diálisis peritoneal, teniendo en cuenta que la tasa de pacientes en tratamiento de DP es inferior al $5 \%{ }^{6}$.

Si la técnica está tecnológicamente preparada, como es el caso; los recursos humanos especializados en D.P. creciendo, aunque no todo lo rápido que se desea; habría que tener en cuenta los criterios de inclusión de dichos pacientes en el programa, pensando además que el paciente, lentamente va adquiriendo más protagonismo en la elección de técnica que se le va a aplicar; en el ámbito de una deseable mayor corresponsabilidad.
Pensamos que es viable incluir a más pacientes en DP, sin olvidar que el sistema también debiera potenciar las dotaciones de personal necesario, las cuales con un menor recurso, podrían proporcionar tratamiento más económico, apoyando así a un sistema sanitario económicamente cada vez más debilitado.

\section{Agradecimientos / Colaboradores:}

Inmaculada Blanco Betorz.

Yolanda Santolaya Ruíz.

Pedro Peñalva Segura.

\section{Bibliografía}

1. Parellada M. Crisis y sostenibilidad del modelo sanitario. Instituto de Economía de Barcelona. Universidad de Barcelona. Abril 2011. Disponible en: http://www. anuariojolyandalucia.com/article/sanidad/930201/ crisis/y/sostenibilidad/modelo/sanitario.html.

2. Sanchéz Bayle M. La sostenibilidad del sistema sanitario público. El País.Abril de2011.Disponibleen:http://www. elpais.com/articulo/sociedad/sostenibilidad/sistema/sanitario/publico/elpepusoc/20100415elpepusoc_2/Tes.

3. Organización Médica Colegial. La OMC recuerda la importancia de que los ciudadanos sigan hábitos de vida saludables para reducir la incidencia de muchas enfermedades crónicas. Disponible en:http://www. noticiasmedicas.es/medicina/noticias/2278/1/LaOMC-recuerda-la-importancia-de-que-los-ciudadanossigan-habitos-de-vida-saludables-para-reducir-la-incidencia-de-muchas-enfermedades-cronicas/Pagel.html.

4. Fernández García E.G. Evaluación de costes en Hemodiálisis. Revista de la Sociedad Española de Nefrología. Noviembre de 2008. Disponible en: http://scielo.isciii. es/pdf/nefro/vllnl/original3.pdf.

5. Oteo Ochoa L.A. Informe: Desarrollo Autonómico, Competitividad y Cohesión Social en el Sistema Sanitario del Consejo Económico y Social, Marzo de 2011. Disponible en: http://my.icloud.com/juherya/webshare/LAO_ Conferencia\%20CES\%2020110311.pdf/LA0_Conferencia\%20CES\%2020110311.pdf.

6. Sociedad Española de Nefrología. Informe de registro de pacientes renales. Año 2009. Disponible en http://www.senefro.org/modules/webstructure/files/ 2reercongsengranada2010.pdf. 